\section{P-101 A RENAL PALLIATIVE MULTIDISCIPLINARY APPROACH}

Louise Gilhooley. Severn Hospice, Shrewsbury, UK

\subsection{6/bmjspcare-2018-hospiceabs. 126}

Background Patients with end stage renal failure on dialysis have a high symptom burden (Murtagh, Addington-Hall \& Higginson, 2007) and survival on dialysis can be comparable to some cancers (Nordio, Limido, Maggiore et al., 2012). Increasingly it is recognised that patients with advancing age and comorbidities may benefit from a conservative approach rather than dialysis (Chandna, Da Silva-Gane, Marshall et al., 2011). Patients with non-malignant disease often receive palliative services late or not at all (Allsop, Ziegler, Mulvey et al., 2018).

Aims To improve renal palliative integration, education and support for patients with End Stage Renal Failure (ESRF), including those on renal replacement therapy and those opting for conservative kidney management.

Methods Development of a monthly renal palliative MDT meeting alongside a biannual two- day renal education programme.

Results From April 2017 to April 2018, 36 patients were identified and included in multidisciplinary discussion. Average age 74 (range 44 to 88 years). Majority included were haemodialysis (HD) patients (27). Six patients had cancer in addition to ESRF. There were 19 deaths during the period. In $71 \%$ of patients who died on HD, dialysis had been withdrawn prior to death. $74 \%$ of patients died at home or in the hospice. In $52 \%$ there was documented advance care planning (escalation decisions and DNAR).

Conclusion A monthly MDT improved access by identifying patients earlier. The referrals have been appropriate and controlled in number. The majority of patients died out of hospital. In our population, HD patients seem to have greater need for referral. Delegates attending the education programme reported an improved confidence in approaching end of life issues and a recognition of the benefits of a collaborative approach. A greater understanding of the population we support and mutual education has resulted in closer working relationships and increased referrals.

Further development of a joint Nephrology/Palliative clinic is planned. A focus group to identify patient and carer needs and experiences is planned.

\section{P-102 INTRODUCING CORNEAL DONATION WITHIN THE HOSPICE SETTING: A QUALITY IMPROVEMENT PROJECT}

Rory Carrigan, Fran Deane, Jo Brady. North London Hospice, London, UK

10.1136/bmjspcare-2018-hospiceabs. 127

Within the UK, there is currently a transplant shortage of approximately 500 corneas each year. Since corneal transplant is a sight-saving procedure, corneal donation (CD) can be empowering for end of life patients who are otherwise unable to donate their organs. Within our organisation, it was felt that more could be done to promote CD.

To achieve this, we invited a specialist team from Moorfields Eye Hospital to train and educate hospice staff on the process of CD. Following this, changes were implemented on the initial patient assessments to facilitate conversations with patients, including a guide for both staff and patients on the process of CD. Staff were also afforded the opportunity to observe the corneal retrieval process to build confidence during discussions with patients.

Prior to our intervention it was unclear what proportion of our patients were engaged in a conversation regarding $C D$, with only one donation occurring in the preceding five years up to November 2017. Between November 2017 and February 2018, CD was discussed with 93 of the 106 patients admitted to the inpatient unit. Four of these patients were referred to Moorfields, resulting in two donations. Reasons for non-donation following referral were (i) contraindication for donation and (ii) a change in the decision to donate following discussions with next of kin.

These preliminary results demonstrate that our intervention has been successful, and data will continue to be collected to confirm this. Guidance on $\mathrm{CD}$ will now be covered in the induction process for new staff to ensure consistency in approaching $\mathrm{CD}$ with patients and their relatives. Future aims include expanding this project into our community teams. To facilitate this, $C D$ champions will be nominated to raise the awareness of CD, ensure that patients' wishes are addressed, and maintain momentum on raising the profile and benefits of CD.

\section{P-103 RECOGNISING DELIRIUM IN HOSPICE INPATIENT UNITS: A QUALITY IMPROVEMENT PROJECT}

Sophie Taylor, Nikki Reed. Marie Curie Hospice, Solihull, UK

10.1136/bmjspcare-2018-hospiceabs. 128

Background Delirium is a common yet serious condition, affecting up to $50 \%$ of all terminally ill patients (Smith \& Adcock, 2011). It can be distressing for patients, carers and staff. Clinical evidence suggests that screening for delirium on admission can help improve outcomes for these patients. However, screening can often be omitted which may adversely affect patient care at the end of life (Baird \& Spiller, 2017).

Aims To ascertain the current level screening for delirium in our hospice inpatient unit. To identify a validated tool for screening for delirium within a hospice setting. To implement the use of a screening tool and formally evaluate its impact on delirium screening rates.

Methods Retrospective case note review to ascertain the current rate of delirium screening on the unit. A literature review to ascertain the most appropriate validated screening tool to be used. A formal quality improvement plan (QIP) using the Plan-Do-Study-Act (PDSA) cycle with the implementation of the valid screening tool.

Results A retrospective case notes review identified that no admissions were being screened for delirium. Initially there was no improvement in screening rates by staff education alone. The literature review highlighted the 4AT as a rapid and user-friendly screening tool. The 4AT screening tool was formally introduced into the admission examination template. By November 2018 we hope to have formal data from our QIP. However, initial data has highlighted the difficulty in sustaining improved rates of screening with a validated tool alone.

Conclusions Screening for delirium on admission to hospice is important to minimise distress at the end of life (Hosker \& Bennett, 2016). Formally implementing the 4AT into our admission process should increase our screening rates. Initial 